\title{
FIXED POINT SETS OF FIBER-PRESERVING MAPS
}

\author{
Robert F. Brown \\ Department of Mathematics \\ University of California \\ Los Angeles, CA 90095 \\ e-mail: rfb@math.ucla.edu \\ Christina L. Soderlund \\ Department of Mathematics \\ California Lutheran University \\ Thousand Oaks, CA 91360 \\ e-mail: csoderlu@callutheran.edu
}

May 22, 2007

\begin{abstract}
Let $\mathfrak{F}=(E, p, B ; Y)$ be a fiber bundle where $E, B$ and $Y$ are connected finite polyhedra. Let $f: E \rightarrow E$ be a fiber-preserving map and $A \subseteq E$ a closed, locally contractible subset. We present necessary and sufficient conditions for $A$ and its subsets to be the fixed point sets of maps fiber-homotopic to $f$. The necessary conditions correspond to those introduced by Schirmer in 1990 but, in the fiber-preserving setting, homotopies are fiber-preserving. Those conditions are shown to be sufficient in the presence of additional hypotheses on the bundle and on the map $f$. The hypotheses can be weakened in the case that $f$ is fiber homotopic to the identity.

Subject Classification 55M20; 55R 10
\end{abstract}

\section{Introduction}

Let $f: X \rightarrow X$ be a self-map of a compact, connected polyhedron. In [10], Schirmer presented necessary and sufficient conditions for a subset $A$ of $X$ to be the set of fixed points Fix $(g)$ of some map $g$ homotopic to $f$. Her results were extended to more general spaces and to maps of pairs in [11]. The purpose of this paper is to investigate Schirmer's problem in the setting of fiber-preserving maps of bundles. 
Schirmer defined a subset $A$ of $X$ to satisfy condition $(C 1)$ for a map $f: X \rightarrow X$ if there exists a homotopy $H_{A}: A \times I \rightarrow X$ from $f \mid A$, the restriction of $f$ to $A$, to the inclusion $i: A \hookrightarrow X$. Condition $(C 2)$ is satisfied if for every essential fixed point class $\mathbb{F}$ of $f$ there exists a path $\alpha: I \rightarrow X$ with $\alpha(0) \in \mathbb{F}, \alpha(1) \in A$ and

$$
\{\alpha(t)\} \backsim\{f \circ \alpha(t)\} *\left\{H_{A}(\alpha(1), t)\right\},
$$

where the symbol $\backsim$ denotes homotopy of paths with endpoints fixed and $*$ the path product.

Schirmer showed that $(C 1)$ and $(C 2)$ are both necessary conditions for realizing $A$ as the fixed point set of a map $g$ homotopic to $f: X \rightarrow X$ ([10], Theorem 2.1). She then invoked the notion of by-passing ([9], Definition 5.1) to prove a sufficiency theorem ([10] Theorem 3.2) using $(C 1)$ and $(C 2)$. A subset $A \subseteq X$ can be bypassed in $X$ if every path in $X$ with endpoints in $X-A$ is homotopic relative to the endpoints to a path in $X-A$.

A topological pair $(X, A)$ will be called a suitable pair if $X$ is a finite polyhedron with no local cut points and $A$ is a closed, locally contractible subspace of $X$ such that $X-A$ is not a 2-manifold and $A$ can be by-passed in $X$. The following is a restatement of Theorem 3.5 of [11] in a form that is convenient for our purposes. It demonstrates that the hypotheses for a suitable pair $(X, A)$ makes it a suitable setting in which to realize sets as fixed point sets.

Theorem 1.1. Let $(X, A)$ be a suitable pair and let $f: X \rightarrow X$ be a map such that $A$ satisfies $(C 1)$ and $(C 2)$ for $f$. If $Z$ is a closed subset of $A$ that intersects every component of $A$, then there exists a map $g$ homotopic to $f$ such that Fix $(g)=Z$.

We will use the term bundle $\mathfrak{F}=(E, p, B ; Y)$ in the sense of [2]. Thus $\mathfrak{F}$ consists of a map $p: E \rightarrow B$, an open cover $\left\{U_{\alpha}\right\}$ of $B$ and a local trivialization consisting of homeomorphisms $\phi_{\alpha}: U_{\alpha} \times Y \rightarrow$ $p^{-1}\left(U_{\alpha}\right)$ such that $p \phi_{\alpha}=\pi$ where $\pi: U_{\alpha} \times Y \rightarrow U_{\alpha}$ is the projection map.

A map $f: E \rightarrow E$ is fiber-preserving with respect to $\mathfrak{F}$ if $e_{1}, e_{2} \in E$ with $p\left(e_{1}\right)=p\left(e_{2}\right)$ implies $p f\left(e_{1}\right)=p f\left(e_{2}\right)$. Thus $f$ induces a map $\bar{f}: B \rightarrow B$ such that $\bar{f} p=p f$. Moreover, if $\bar{f}(b)=b$, then the restriction of $f$ to $p^{-1}(b)$ is a map $f_{b}: p^{-1}(b) \rightarrow p^{-1}(b)$. A homotopy $H: E \times I \rightarrow E$ is fiber-preserving if each map $h_{t}: E \rightarrow E$ defined by $h_{t}(e)=H(e, t)$, for $t \in I$, is fiber-preserving, and then $h_{0}$ is said to be fiber homotopic to $h_{1}$, written $h_{0} \simeq_{\mathfrak{F}} h_{1}$. A fiber homotopy $H: E \times I \rightarrow E$ induces a homotopy $\bar{H}: B \times I \rightarrow B$.

Given a bundle $\mathfrak{F}=(E, p, B ; Y)$ and a fiber-preserving map $f: E \rightarrow E$ we will investigate conditions on a locally contractible 
subset $A \subseteq E$ so that there is a map $g: E \rightarrow E$ fiber homotopic to $f$ with Fix $(g)=A$. We next present a simple example in which $A$ is the fixed point set of a map $g$ that is homotopic to a fiber-preserving map $f: E \rightarrow E$ but $A$ cannot be the fixed point set of any map that is fiber homotopic to $f$. This example illustrates the fact that the characterization of fixed point sets for a homotopy class becomes significantly different in the fiber-preserving setting.

Example 1.1. Consider the fiber space $\mathfrak{F}=(E, p, B)$ in which $E=$ $S^{1} \times S^{1}=T^{2}$, the 2-dimensional torus, $B=S^{1}$ and $p: E \rightarrow B$ is the projection map onto the first coordinate. We represent points of $T^{2}$ as pairs $\left(e^{i 2 \pi s}, e^{i 2 \pi t}\right)$ and define a fiber-preserving map $f: T^{2} \rightarrow T^{2}$ by

$$
f\left(e^{i 2 \pi s}, e^{i 2 \pi t}\right)=\left(e^{i 2 \pi s}, e^{i 2 \pi(3 t)}\right) .
$$

Let $A=S^{1} \times\{1\}$. The map $g: T^{2} \rightarrow T^{2}$ defined by

$$
g\left(e^{i 2 \pi s}, e^{i 2 \pi t}\right)=\left(e^{i 2 \pi\left(s+t-t^{2}\right)}, e^{i 2 \pi(3 t)}\right) .
$$

is homotopic to $f$ and $\operatorname{Fix}(g)=A$. However, any fiber-preserving map that is fixed on $A$ must take each fiber to itself. If such a map were fiber homotopic to $f$, then its degree on each fiber would be the same as that of $f$, that is three, and therefore it must have at least two fixed points on each fiber, so $A$ could not be the entire fixed point set.

In the next section, we present the fiber-preserving analogues of Schirmer's conditions (C1) and (C2) and show that, given a fiberpreserving map $f: E \rightarrow E$ of a bundle $\mathfrak{F}$, they are necessary conditions on a subset $A$ of $E$ for the existence of a map $g$ fiber homotopic to $f$ such that $\operatorname{Fix}(g)=A$. Section 3 is devoted to bundle constructions that we use in Section 4 to obtain sufficient conditions for the existence of such a map $g$. In Section 5 we discuss another approach to sufficiency that applies to a class of fiber deformations, that is, maps fiber homotopic to the identity.

We thank Ed Fadell, of whom the first author is a student and the second a grand-student, for sharing with us his insights concerning the topology of bundles. We also thank the referee of the paper who wrote a thorough and thought-provoking report.

\section{Necessary Conditions}

Throughout the paper, we will assume that, for the bundle $\mathfrak{F}=$ $(E, p, B ; Y)$, the spaces $E, B$ and $Y$ are connected finite polyhedra. 
We give here two conditions, analogous to Schirmer's conditions (C1) and (C2), that any subset $A$ of the total space $E$ must satisfy if we wish to realize $A$ as the fixed point set of a map fiber homotopic to a given fiber-preserving map $f: E \rightarrow E$. Later we will show that they are sufficient when appropriate hypotheses are added.

Definition. We say that $A$ satisfies conditions $\left(C 1_{\mathfrak{F}}\right)$ and $\left(C 2_{\mathfrak{F}}\right)$ for a given fiber-preserving map $f: E \rightarrow E$ if the following are satisfied:

$\left(C 1_{\mathfrak{F}}\right)$ There exists a fiber-preserving homotopy $H_{A}: A \times I \rightarrow E$ from $f \mid A$ to the inclusion $i: A \hookrightarrow E$,

$\left(C 2_{\mathfrak{F}}\right)$ for every essential fixed point class $\mathbb{F}$ of $f$, there exists a path $\alpha: I \rightarrow E$ with $\alpha(0) \in \mathbb{F}, \alpha(1) \in A$ and

$$
\{\alpha(t)\} \sim\{f \circ \alpha(t)\} *\left\{H_{A}(\alpha(1), t)\right\},
$$

where $H_{A}$ is the fiber-preserving homotopy from $\left(C 1_{\mathfrak{F}}\right)$.

Thus, for both conditions, the only change is that the homotopy $H_{A}$ must be fiber-preserving.

Theorem 2.1. (Necessity) Let $\mathfrak{F}=(E, p, B ; Y)$ be a bundle where $E, B$ and $Y$ are connected finite polyhedra. Let $f: E \rightarrow E$ be a fiber-preserving map and let $A$ be a subspace of $E$. If there exists a map $g$ fiber-homotopic to $f$ with Fix $(g)=A$, then $A$ satisfies $\left(C 1_{\mathfrak{F}}\right)$ and $\left(C 2_{\mathfrak{F}}\right)$ for $f$.

Proof. Let $H: E \times I \rightarrow E$ denote the fiber-preserving homotopy from $f$ to $g$ and let $H_{A}=H \mid A$ be the restriction of $H$ to $A$. To verify $\left(C 1_{\mathfrak{F}}\right)$, we must show that $H_{A}$ is a fiber-preserving homotopy from $f \mid A$ to $i: A \hookrightarrow E$. It is clear that $H_{A}$ is a homotopy from $f \mid A$ to $i$. To see that it is also fiber-preserving, recall that since $H$ is fiber-preserving, we have

$$
p \circ H=\bar{H} \circ(p \times i d)
$$

for $\bar{H}: B \times I \rightarrow B$. Thus by restricting to $A$,

$$
\begin{aligned}
p \circ H \mid A & =\left.\bar{H} \circ(p \times i d)\right|_{(A \times I)} \\
\Rightarrow p \circ H_{A} & =\bar{H} \circ(p \mid A \times i d),
\end{aligned}
$$

and we see that $\left(C 1_{\mathfrak{F}}\right)$ holds.

To verify $\left(C 2_{\mathfrak{F}}\right)$, choose any essential fixed point class $\mathbb{F}$ of $f$. Then there exists a unique essential fixed point class $\mathbb{G}$ of $g$ that is H-related to $\mathbb{F}$. In particular, we can find a path $\alpha: I \rightarrow E$ with $\alpha(0) \in \mathbb{F}, \alpha(1) \in \mathbb{G}$ and 


$$
\begin{aligned}
\{\alpha(t)\} & \sim\{H(\alpha(t), t)\} \\
& \sim\{H(\alpha(t), 0)\} *\{H(\alpha(1), t)\} \\
& =\{f \circ \alpha(t)\} *\left\{H_{A}(\alpha(1), t)\right\},
\end{aligned}
$$

where the equality follows from the definitions of $H$ and $H_{A}$.

The following lemma provides a version of condition $(C 2)$ on the base space $B$ that we will use in the next section.

Lemma 2.1. Let $\mathfrak{F}=(E, p, B ; Y)$ be a bundle where $E, B$ and $Y$ are connected finite polyhedra and let $f: E \rightarrow E$ be a fiber-preserving map. Suppose $A \subseteq E$ satisfies $\left(C 1_{\mathfrak{F}}\right)$ and $\left(C 2_{\mathfrak{F}}\right)$ for $f$. Then for every essential fixed point class $\mathbb{F}$ of $f$ there exists a path $\bar{\alpha}: I \rightarrow B$ with $\bar{\alpha}(0) \in p(\mathbb{F}), \bar{\alpha}(1) \in p(A)$ and

$$
\{\bar{\alpha}(t)\} \backsim\{\bar{f} \circ \bar{\alpha}(t)\} *\left\{\bar{H}_{A}(p \circ \alpha(1), t)\right\} .
$$

Proof. Choose any essential fixed point class $\mathbb{F}$ of $f$. From $\left(C 2_{\mathfrak{F}}\right)$, there exists a path $\alpha: I \rightarrow E$ with $\alpha(0) \in \mathbb{F}, \alpha(1) \in A$ and

$$
\{\alpha(t)\} \sim\{f \circ \alpha(t)\} *\left\{H_{A}(\alpha(1), t)\right\} .
$$

Since $f$ and $H_{A}$ are both fiber-preserving, we have

$$
\begin{aligned}
\{p \circ \alpha(t)\} & \backsim\{p \circ f \circ \alpha(t)\} *\left\{p \circ H_{A}(\alpha(1), t)\right\} \\
& =\{\bar{f} \circ p \circ \alpha(t)\} *\left\{\bar{H}_{A}(p \circ \alpha(1), t)\right\},
\end{aligned}
$$

from the definitions of $\bar{f}$ and $\bar{H}$. Setting $\bar{\alpha}=p \circ \alpha$, we obtain

$$
\{\bar{\alpha}(t)\} \backsim\{\bar{f} \circ \bar{\alpha}(t)\} *\left\{\bar{H}_{A}(p \circ \alpha(1), t)\right\} .
$$

\section{The Construction Over $B-p(A)$}

For any self-map $f$ on a compact, connected polyhedron, a set $\mu \subseteq$ Fix $(f)$ is called a set of essential representatives of $f$ if $\mu$ contains exactly one point from every essential fixed point class of $f$ ([3], Definition 4.1).

Definition. ([4], Def. 6.1) Let $f$ be a fiber-preserving map for $\mathfrak{F}=$ $(E, p, B ; Y)$. The fiberwise Nielsen number $N_{\mathfrak{F}}(f, p)$ of $f$ is defined by

$$
N_{\mathfrak{F}}(f, p)=\sum_{b \in \mu} N\left(f_{b}\right),
$$

where $\mu$ is any set of essential representatives of $\bar{f}$. 
The purpose of this section is to prove

Theorem 3.1. Let $\mathfrak{F}=(E, p, B ; Y)$ be a bundle where $E, B$ and $Y$ are connected finite polyhedra and let $f: E \rightarrow E$ be a fiber-preserving map. Suppose $A$ is a closed subset of $E$ such that $(B, p(A))$ is a suitable pair and $A$ satisfies $\left(C 1_{\mathfrak{F}}\right)$ and $\left(C 2_{\mathfrak{F}}\right)$ for $f$, then there exists a map $g: E \rightarrow E$ fiber-homotopic to $f$ such that

$$
A \subseteq F i x(g) \subseteq p^{-1}(p(A))
$$

and Fix $(\bar{g}) \cap(B-p(A))$ is finite.

Proof. First let us consider the case when $A=\emptyset$. As $\left(C 2_{\mathfrak{F}}\right)$ holds for $A$, and no essential fixed point class can be connected to $\emptyset$ by a path, we know that $N(f)=0$. Consequently, $N_{\mathfrak{F}}(f, p)=0$ ([4], Proposition 7.3(1)). Next observe that the base space $B$ satisfies the hypotheses of Theorem 5.3 of [6]. Thus, there exists a map $\bar{g}$ homotopic to $\bar{f}$ with $N(\bar{f})$ fixed points. This implies that the fiber map $f$ is fiber homotopic to a map with $N_{\mathcal{F}}(f, p)=0$ fixed points ([4], Theorem 8.2), which concludes the proof for $A=\emptyset$.

Suppose $A \neq \emptyset$, then $p(A)$ is a nonempty closed subset of $B$. If we let $H_{A}: A \times I \rightarrow E$ denote the fiber-preserving homotopy from $\left(C 1_{\mathfrak{F}}\right)$, then the induced map $\bar{H}_{A}: p(A) \times I \rightarrow B$ is a homotopy from $\bar{f} \mid p(A)$ to $i_{p(A)}: p(A) \hookrightarrow B$. Thus, $\bar{f}$ satisfies condition $(C 1)$ for $p(A)$. We will prove the theorem in three steps.

Step 1. We will show that there exists a homotopy $\bar{H}: B \times I \rightarrow B$, extending $\bar{H}_{A}$, from $\bar{f}$ to a map $\bar{u}: B \rightarrow B$ such that

(i) Fix $(\bar{u})=p(A) \cup\left\{b_{1}, b_{2}, \ldots, b_{r}\right\}$,

(ii) each $b_{j}$ forms an essential fixed point class of $\bar{u}$ with the property that $\left\{b_{j}\right\}$ is not $\bar{H}$-related to any essential fixed point class of $\bar{f}$ that lies in the image under $p$ of any essential fixed point class of $f$.

Consider any essential fixed point class $\overline{\mathbb{F}}$ of $\bar{f}$ satisfying $\overline{\mathbb{F}}=p(\mathbb{F})$ for some essential fixed point class $\mathbb{F}$ of $f$. The map $\bar{f}$ satisfies $(C 2)$ for $p(A)$ with respect to this class $\overline{\mathbb{F}}$ by Lemma 2.1 .

We can apply the homotopy extension property to extend $\bar{H}_{A}$ to a homotopy $\bar{H}_{1}: B \times I \rightarrow B$ and define a map $\bar{u}_{1}: B \rightarrow B$ by $\bar{u}_{1}(x)=\bar{H}_{1}(x, 1)$. By the proof of Theorem 4.1 of [9], there exists a star cover $K$ of $p(A)$ and a map $\bar{u}_{2}$ homotopic to $\bar{u}_{1}$ such that $\operatorname{Fix}\left(\bar{u}_{2}\right) \cap(\operatorname{St}(p(A))-p(A))=\emptyset$, the fixed point set of $u_{2}$ contains $p(A)$ and $\bar{u}_{2}$ is fix-finite on $B-\operatorname{St}(p(A))$. 
By the proof of Lemma 3.1 of [10], we can find a map $\bar{u}: B \rightarrow B$ homotopic to $\bar{u}_{2}$ (and hence homotopic to $\bar{f}$ ) with the property that $p(A) \subseteq$ Fix $\bar{u}$ and every point $b_{j} \in \operatorname{Fix}(\bar{u})-p(A)$ forms an essential fixed point class $\left\{b_{j}\right\}$ of $\bar{u}$. Let $\bar{H}: B \times I \rightarrow B$ denote the homotopy from $\bar{f}$ to $\bar{u}$. Then $\bar{H}$ extends $\bar{H}_{A}$ by the proof of Lemma 3.1 of [10]. Further, every $b_{j} \in \operatorname{Fix}(\bar{u})-p(A)$ is $\bar{H}$-related to an essential fixed point class $\overline{\mathbb{F}}$ of $\bar{f}$.

Now if this $\overline{\mathbb{F}}$ lies in the image under $p$ of an essential fixed point class $\mathbb{F}$ of $f$, then we have $\bar{\alpha}$ as in Lemma 2.1

$$
\begin{aligned}
\{\bar{\alpha}(t)\} & \backsim\{\bar{f} \circ \bar{\alpha}(t)\} *\left\{\bar{H}_{A}(\bar{\alpha}(1), t)\right\} \\
& \backsim\{\bar{H}(\bar{\alpha}(t), 0)\} *\{\bar{H}(\bar{\alpha}(1), t)\} \\
& \backsim\{\bar{H}(\bar{\alpha}(t), t)\},
\end{aligned}
$$

because $\bar{H}$ extends $\bar{H}_{A}$.

Then by $\bar{H}$-relatedness, $\bar{\alpha}(1) \in\left\{b_{j}\right\}$. Since $b_{j}$ is the only fixed point in its class, $\bar{\alpha}(1)=b_{j}$. But $\bar{\alpha}(1) \in p(A)$, contradicting our assumption that $b_{j} \notin p(A)$. Thus, there are no points $b_{j} \in \operatorname{Fix}(\bar{u})-$ $p(A)$ that are $\bar{H}$-related to $p(\mathbb{F})$ for any essential fixed point class $\mathbb{F}$ of $f$. This completes Step 1 .

Step 2. We will show that $f$ is fiber homotopic to a map $v: E \rightarrow$ $E$ with $\operatorname{Fix}(v) \subseteq p^{-1}(p(A))$.

By the homotopy lifting property, we can find a fiber-preserving homotopy $H: E \times I \rightarrow E$ lifting $\bar{H}$ where for each $e \in E, H(e, 0)=$ $f(e)$. We define $u(e)=H(e, 1)$. Then $u$ maps every fiber in $p^{-1}(\operatorname{Fix}(\bar{u}))$ to itself and moves every other fiber in $E$. In particular,

$$
\operatorname{Fix}(u) \subseteq p^{-1}(\operatorname{Fix}(\bar{u}))=p^{-1}\left(p(A) \cup \bigcup_{j=1}^{r} b_{j}\right) .
$$

For each point $b_{j} \in \operatorname{Fix}(\bar{u})-p(A)$, consider the restriction of $u$ to $p^{-1}\left(b_{j}\right)$ which we denote by $u_{b_{j}}: p^{-1}\left(b_{j}\right) \rightarrow p^{-1}\left(b_{j}\right)$. We will show that $N\left(u_{b_{j}}\right)=0$. To obtain a contradiction, we suppose there is an essential fixed point class of $u_{b_{j}}$ and choose any point $e_{b_{j}} \in p^{-1}\left(b_{j}\right)$ lying in this class. By the construction in Step 1, $p\left(e_{b_{j}}\right)=b_{j}$ is an essential fixed point class of $\bar{u}$. This implies that $e_{b_{j}}$ must also lie in an essential fixed point class of $u$ ([13], Theorem 4.1). As $u$ is fiber-homotopic to $f$, this essential fixed point class must be $H$-related to an essential fixed point class of $f$. But this implies that their images under $p$ are $\bar{H}$-related, contradicting Step 1 (ii) for $b_{j}$. Therefore $N\left(u_{b_{j}}\right)=0$ and hence $u_{b_{j}}$ is homotopic to a fixed 
point free map on the fiber ([6], Theorem 5.3). Denote this map by $v_{b_{j}}: p^{-1}\left(b_{j}\right) \rightarrow p^{-1}\left(b_{j}\right)$.

We wish to extend the homotopy between the $u_{b_{j}}$ and $v_{b_{j}}$ to the total space $E$. We consider the map $G: E \times\{0\} \cup\left(p^{-1}(\operatorname{Fix}(\bar{u})) \times I \rightarrow\right.$ $E$ defined by $G(e, 0)=u(e), G(e, t)=u(e)$ if $p(e) \in A$ and, on $p^{-1}\left(b_{j}\right)$, let $G(e, t)$ be the homotopy from $u_{b_{j}}$ to $v_{b_{j}}$. Then, by the fiber homotopy extension theorem ([1], Theorem 2.1), we can extend $G$ to a homotopy $G: E \times I \rightarrow E$. The map $v: E \rightarrow E$ is defined by $v(e)=G(e, 1)$. We note that, by construction, the map of $B$ induced by $v$ is $\bar{u}$.

Step 3. We will show that $f$ is fiber-homotopic to a map $g: E \rightarrow$ $E$ with $A \subseteq \operatorname{Fix}(g) \subseteq p^{-1}(p(A))$.

Since $\left.f\right|_{A} \simeq_{\mathcal{F}} i_{A}$ from $\left(C 1_{\mathcal{F}}\right)$ and $v \simeq_{\mathcal{F}} f$, we can construct a fiber-preserving homotopy $J: A \times I \rightarrow E$ from $\left.v\right|_{A}$ to $i_{A}$. For $j=1, \ldots, r$ and $e \in p^{-1}\left(b_{j}\right)$, define $J(e, t)=v_{b_{j}}(e)$ for all $t$. With another application of the fiber homotopy extension theorem, we extend $J$ to a homotopy $H: E \times I \rightarrow E$ satisfying $H(e, 0)=v(e)$ for any $e \in E$. Define the map $g: E \rightarrow E$ by $g(e)=H(e, 1)$ for $e \in E$.

Observe that $\left.g\right|_{A}=i_{A}$ by construction, thus, $A \subseteq \operatorname{Fix}(g)$. Also, as all homotopies have been fiber-preserving, and as all fixed fibers outside $A$ have no fixed points, $\operatorname{Fix}(g) \subseteq p^{-1}(p(A))$.

Corollary 3.1. Let $\mathfrak{F}=(E, p, B ; Y)$ be a bundle where $E, B$ and $Y$ are finite polyhedra. Suppose $A$ is a closed subset of $E$ such that $p^{-1}(p(A))=A$ and $(B, p(A))$ is a suitable pair. If $A$ satisfies $\left(C 1_{\mathfrak{F}}\right)$ and $\left(C 2_{\mathfrak{F}}\right)$ for a fiber-preserving map $f: E \rightarrow E$, then there exists a map $g: E \rightarrow$ E fiber-homotopic to $f$ such that Fix $(g)=A$.

\section{Sufficient Conditions}

The difficulty in making constructions in bundles comes from the fact that, if $U_{\alpha}$ and $U_{\beta}$ are in the locally trivializing cover of $B$, then $p^{-1}\left(U_{\alpha} \cap U_{\beta}\right)$ has two different trivializations given by restrictions of $\phi_{\alpha}$ and $\phi_{\beta}$. Thus, for instance, local cross-sections may not combine into a global cross-section, as obstruction theory demonstrates. In the extensive literature of the fixed point theory of a fiber-preserving map $f: E \rightarrow E$, it has been customary to first homotope $\bar{f}: B \rightarrow B$ to a map with finitely many fixed points and then use the homotopy lifting property to produce a map that is fiber homotopic to $f$ and can have fixed points in only finitely many 
fibers. Neighborhoods of those isolated fibers can be given trivializations without concern for the overlapping trivialization problem that occurs in constructing cross-sections. Since, for the problem of realizing a subset $A$ of $X$ as the fixed point set of a map homotopic to a given fiber-preserving map we cannot change $A$, we need to impose an appropriate condition. Of course the requirement that $p(A)$ is finite would allow us to proceed, and that is an interesting special case, but we can employ a less restrictive condition.

Let $\mathfrak{F}=(E, p, B ; Y)$ be a bundle, so we have an open cover $\left\{U_{\alpha}\right\}$ of $B$ and homeomorphisms $\phi_{\alpha}: U_{\alpha} \times Y \rightarrow p^{-1}\left(U_{\alpha}\right)$ such that $p \phi_{\alpha}=$ $\pi$. A topological pair $\left(E, E_{0}\right)$ is a bundle pair (see [2], page 440) with respect to the bundle $\mathfrak{F}=(E, p, B ; Y)$ if there is a nonempty subspace $Y_{0}$ of $Y$ such that the restriction of each local trivialization $\phi_{\alpha}$ of $\mathfrak{F}$ to $U_{\alpha} \times Y_{0}$ is a homeomorphism onto $p^{-1}\left(U_{\alpha}\right) \cap E_{0}$. Thus we have a bundle $\mathfrak{F}_{0}=\left(E_{0}, p, B ; Y_{0}\right)$ with respect to the same cover $\left\{U_{\alpha}\right\}$ and the restrictions of the $\phi_{\alpha}$.

Lemma 4.1. Let $\left(\mathfrak{F}, \mathfrak{F}_{0}\right)=\left(\left(E, E_{0}\right), p, B ;\left(Y, Y_{0}\right)\right)$ be a bundle pair where $E, B$ and $Y$ are connected finite polyhedra, $E_{0}$ is a closed, locally contractible subset of $E$ and $B$ is contractible. Let $f: E \rightarrow E$ be a fiber-preserving map such that $p f=p$, that is, $f$ maps each fiber to itself, and $E_{0} \subseteq$ Fix $(f)$. Suppose $\left(Y, Y_{0}\right)$ is a suitable pair and $E_{0}$ intersects every essential fixed point class of $f_{b_{0}}: p^{-1}\left(b_{0}\right) \rightarrow p^{-1}\left(b_{0}\right)$ for some $b_{0} \in B$. If $\left(E_{0}, Z\right)$ is a bundle pair such that $Z$ is a closed subset of $E_{0}$ that intersects all of the components of $E_{0}$, then there exists a map $g: E \rightarrow E$ that is fiber homotopic to $f$ such that $F i x(g)=Z$.

Proof. Since a bundle with contractible base is trivial ([12], Cor. 11.6, page 53), we have a homeomorphism $\phi: B \times Y \rightarrow E$ such that $\phi\left(B \times Y_{0}\right)=E_{0}$ and $p \phi=\pi$. Define $f^{*}=\phi^{-1} f \phi: B \times Y \rightarrow$ $B \times Y$ and note that $\pi f^{*}=\pi$. Thus we may write $f^{*}$ in the form $f^{*}(b, y)=\left(b, f_{b}^{*}(y)\right)$. Since $B$ is contractible, there is a homotopy $K: B \times I \rightarrow B$ such that, for all $b \in B$, we have $K(b, 0)=b$ and $K(b, 1)=b^{\prime}$, for some $b^{\prime} \in B$. Define $U^{*}:(B \times Y) \times I \rightarrow B \times Y$ by

$$
U^{*}((b, y), t)= \begin{cases}\left(b, f_{K(b, 2 t)}^{*}(y)\right) & 0 \leq t \leq \frac{1}{2} \\ \left(b, f_{K\left(b_{0}, 2-2 t\right)}^{*}(y)\right) & \frac{1}{2} \leq t \leq 1,\end{cases}
$$

where, by hypothesis, $b_{0} \in B$ has the property that every essential fixed point class of $f_{b_{0}}$ is intersected by $E_{0}$. Then $U^{*}((b, y), 0)=$ $\left(b, f_{b}^{*}(y)\right)=f^{*}(b, y)$ and $U^{*}((b, y), 1)=\left(b, f_{b_{0}}^{*}(y)\right)$ so $f^{*}$ is fiber homotopic to $i d \times f_{b_{0}}^{*}$, where $i d$ denotes the identity map of $B$. If $(b, y) \in B \times Y_{0}$, then $\phi(b, y) \in E_{0}$ so $E_{0} \subseteq \operatorname{Fix}(f)$ implies 
that $f^{*}(b, y)=(b, y)$ and thus $B \times Y_{0} \subseteq \operatorname{Fix}\left(f^{*}\right)$. In particular, $Y_{0} \subseteq \operatorname{Fix} f_{b}^{*}$ for each $b \in B$, and thus $Y_{0} \subseteq \operatorname{Fix}\left(f_{b_{0}}^{*}\right)$. By the commutativity property of the fixed point index, $\phi^{-1}$ determines a oneto-one correspondence between the essential fixed point classes of $f_{b_{0}}$ and those of $f_{b_{0}}^{*}$ (see [7], page 20). By hypothesis, each essential class of $f_{b_{0}}$ is intersected by $E_{0}$ and thereby also intersected by $E_{0} \cap p^{-1}\left(b_{0}\right)$. Therefore, since $\phi^{-1}\left(E_{0} \cap p^{-1}\left(b_{0}\right)\right)=b_{0} \times Y_{0}$, the oneto-one correspondence implies that $Y_{0}$ intersects all essential fixed point classes of $f_{b_{0}}^{*}$.

Thus, by Theorem 4.2(ii) of [10], $Y_{0}$ satisfies conditions $(\mathrm{C} 1)$ and $(\mathrm{C} 2)$ for $f_{b_{0}}^{*}$. For the bundle pair $\left(\mathfrak{F}_{\mathfrak{o}}, \mathfrak{Z}\right)=\left(\left(E_{0}, Z\right), p, B ;\left(Y_{0}, \zeta\right)\right)$, the set $Z$ intersects every component of $E_{0}$ by hypothesis and $E_{0}$ is homeomorphic to $B \times Y_{0}$, so $\phi^{-1}(Z)$ intersects every component of $b_{0} \times Y_{0}$ and hence $\zeta$ intersects every component of $Y_{0}$. Therefore the hypotheses of Theorem 1.1 are satisfied and there is a map $g_{b_{0}}^{*}: Y \rightarrow$ $Y$, homotopic to $f_{b_{0}}^{*}$ by a homotopy we denote by $V^{*}: Y \times I \rightarrow Y$, such that $\operatorname{Fix}\left(g_{b_{0}}^{*}\right)=\zeta$. Define $H^{*}:(B \times Y) \times I \rightarrow B \times Y$ by

$$
H^{*}((b, y), t)= \begin{cases}U^{*}((b, y), 2 t) & 0 \leq t \leq \frac{1}{2} \\ \left(b, V^{*}(y, 2-2 t)\right) & \frac{1}{2} \leq t \leq 1\end{cases}
$$

Thus $f^{*}$ is fiber homotopic to $i d \times g_{b_{0}}^{*}$ where, we note, $\operatorname{Fix}\left(i d \times g_{b_{0}}^{*}\right)=$ $B \times \zeta$. Now define $H: E \times I \rightarrow E$ by

$$
H(e, t)=\phi\left(H^{*}\left(\phi^{-1}(e), t\right)\right)
$$

then $H(e, 0)=f(e)$ and, for $g$ defined by $g(e)=H(e, 1)$, we have $\operatorname{Fix}(g)=\phi(B \times \zeta)=Z$.

The requirement that $Z$ be a bundle allows us to apply Theorem 1.1 to obtain a homotopy on a single fiber and then extend that homotopy to all of $E$. The restrictions on $Z$ are otherwise very mild since it need only be a closed subset of $E_{0}$ that intersects all of its components.

In order to take advantage of the local product structure of bundles yet allow our fixed point sets sufficient generality to include a variety of examples, we will generalize the bundle pair concept. We will use the concept of the restriction of $\mathfrak{F}=(E, p, B ; Y)$ to $W \subseteq B$ which is defined to be the bundle $\mathfrak{F} \mid W=\left(p^{-1}(W), p, W ; Y\right)$ where the local trivialization $\phi_{\alpha}:\left(U_{\alpha} \cap W\right) \times Y \rightarrow p^{-1}\left(U_{\alpha} \cap W\right)$ is the restriction of $\phi_{\alpha}$ (compare [5], Def. 5.1, page 17). We will say that a subset $A$ of $E$ is a bundle subset of the bundle $\mathfrak{F}$ if, for each component $p(A)_{j}$ of $p(A)$, the pair $\left(p^{-1}\left(p(A)_{j}\right), A_{j}\right)$, where $A_{j}=A \cap p^{-1}\left(p(A)_{j}\right)$, is a bundle pair with respect to the restriction bundle $\mathfrak{F} \mid p(A)_{j}$. 
Note that the definition of bundle subset allows for the possibility that, for the various components of $p(A)_{j}$, there may be different subbundle fibers $Y_{j} \subseteq Y$. Example 4.6 of [4] describes fiberpreserving maps whose fixed point sets are of this type. The bundle is the standard fibration $p: K^{2} \rightarrow S^{1}$ of the Klein bottle over the circle. Given an odd integer $r$ and an integer $q$, a map $f$ is defined with the property that $\operatorname{Fix}(\bar{f})$ consists of $|r-1|$ points . In the fiber over half the points of $\operatorname{Fix}(\bar{f})$, the map $f$ has $|1-q|$ fixed points whereas, in the fiber over every one of the other points of $\operatorname{Fix}(\bar{f})$, the number of fixed points of $f$ is $|1+q|$.

Theorem 4.1. Let $\mathfrak{F}=(E, p, B ; Y)$ be a bundle where $E, B$ and $Y$ are connected finite polyhedra, let $f: E \rightarrow E$ be a fiber-preserving map and let $A$ be a closed, locally contractible subset of $E$ that is a bundle subset of $E$ such that each component $p(A)_{j}$ of $p(A)$ is contractible and $(B, p(A))$ and $\left(Y, Y_{j}\right)$, for all subbundle fibers $Y_{j}$ of $A$, are suitable pairs. Suppose $A$ satisfies $\left(C 1_{\mathfrak{F}}\right)$ and $\left(C 2_{\mathfrak{F}}\right)$ for $f$ and $A$ intersects every essential fixed point class of $f_{b_{j}}: p^{-1}\left(b_{j}\right) \rightarrow p^{-1}\left(b_{j}\right)$ for at least one $b_{j}$ in each component $p(A)_{j}$. If $Z$ is a closed bundle subset of $A$ that intersects every component of $A$, then there exists a map $g: E \rightarrow E$ that is fiber homotopic to $f$ such that Fix $(g)=Z$.

Proof. By Theorem 3.1, we may assume that

$$
A \subseteq F i x(f) \subseteq p^{-1}(p(A))
$$

and $\bar{f}: B \rightarrow B$ has a finite set $F$ of fixed points on $B-p(A)$. By the proof of the same theorem, we may also assume $A \neq \emptyset$. Let $f_{j}: p^{-1}\left(p(A)_{j}\right) \rightarrow p^{-1}\left(p(A)_{j}\right)$ be the restriction of $f$ and note that $p f_{j}=p$. Since the hypotheses of Lemma 4.1 are satisfied for the bundle pairs $\left(p^{-1}\left(p(A)_{j}\right), A_{j}\right)$ and $\left(A_{j}, Z_{j}\right)$, where $Z_{j}=Z \cap A_{j}$, there is a fiber-preserving homotopy $H_{j}: p^{-1}\left(p(A)_{j}\right) \times I \rightarrow p^{-1}\left(p(A)_{j}\right)$ such that $H_{j}(e, 0)=f_{j}(e)$ and, for $g_{j}$ defined by $g_{j}(e)=H_{j}(e, 1)$, we have $\operatorname{Fix}\left(g_{j}\right)=Z_{j}$. Let

$$
H: E \times\{0\} \cup\left(p^{-1}(F) \cup p^{-1}(p(A))\right) \times I \rightarrow E
$$

be defined by

$$
H(e, t)= \begin{cases}f(e) & t=0 \text { or } p(e) \in F \\ H_{j}(e, t) & p(e) \in p(A)_{j}\end{cases}
$$

By the fiber homotopy extension theorem, $H$ can be extended to a map $G: E \times I \rightarrow E$ such that for each $t, p G(e, t)=\bar{f} p(e)$. Defining $g: E \rightarrow E$ by $g(s)=G(e, 1)$ gives us a map that is fiber homotopic to $f$ and $F i x(g)=Z$. 
Corollary 4.1. Let $\mathfrak{F}=(E, p, B ; Y)$ be a bundle where $E, B$ and $Y$ are finite polyhedra, let $f: E \rightarrow E$ be a fiber-preserving map and let $A$ be a closed, locally contractible subset of $E$ such that $p(A)=$ $\left\{b_{1}, \ldots, b_{r}\right\}$ and $(B, p(A))$ and all $\left(p^{-1}\left(b_{j}\right), A_{j}\right)$ are suitable pairs. Suppose $A$ satisfies $\left(C 1_{\mathfrak{F}}\right)$ and $\left(C 2_{\mathfrak{F}}\right)$ for $f$ and $A_{j}$ intersects every essential fixed point class of $f_{b_{j}}: p^{-1}\left(b_{j}\right) \rightarrow p^{-1}\left(b_{j}\right)$, for $j=1, \ldots, r$. If $Z$ is a closed subset of $A$ that intersects every component of $A$, then there exists a map $g: E \rightarrow E$ that is fiber-homotopic to $f$ such that Fix $(g)=Z$.

\section{$5 \quad$ Fiber deformations}

For $S$ a subset of a space $X$, we introduce the notation $S^{c}$ to denote the closure of the complement $X-S$ of $S$. For a bundle pair $\left(\mathfrak{F}, \mathfrak{F}_{0}\right)=\left(\left(E, E_{0}\right), p, B ;\left(Y, Y_{0}\right)\right)$, we define the corresponding complement bundle $\mathfrak{F}_{0}^{c}=\left(E_{0}^{c}, p_{c}, B ; Y_{0}^{c}\right)$ where $p_{c}$ is the restriction of $p$. Restricting $\phi_{\alpha}$ gives the local trivialization $U_{\alpha} \times Y_{0}^{c} \rightarrow p_{c}^{-1}\left(U_{\alpha}\right)$ that makes $\mathfrak{F}_{0}^{c}$ a bundle with respect to the cover $\left\{U_{\alpha}\right\}$ of $B$ that was used for both $\mathfrak{F}$ and $\mathfrak{F}_{0}$.

We will say that a bundle pair $\left(\mathfrak{F}, \mathfrak{F}_{0}\right)$ has trivial complement if the corresponding complement bundle $\mathfrak{F}_{0}^{c}$ is trivial, that is, there is a homeomorphism $\phi: B \times Y_{0}^{c} \rightarrow E_{0}^{c}$ such that $p_{c} \phi=\pi$. The bundle pair $\left(\mathfrak{F}, \mathfrak{F}_{0}\right)$ has trivial complement if $\mathfrak{F}$ is a trivial bundle, but bundle pairs with trivial complement are not limited to trivial bundle pairs.

For an example where neither $\mathfrak{F}$ nor $\mathfrak{F}_{0}$ is trivial but they have trivial complement, let the Klein bottle $K^{2}$ be represented as the quotient space of $[-1,1] \times[-1,1]$ under the equivalence relation $(s,-1) \sim(s, 1)$ and $(-1, t) \sim(1,-t)$. Then projection on the first factor gives a nontrivial bundle $\mathfrak{F}_{0}=\left(E_{0}=K^{2}, p, S^{1} ; S^{1}\right)$. Now represent the torus $T^{2}=S^{1} \times S^{1}$ as $[-1,1] \times[-1,1]$ under the equivalence relation $(s,-1) \sim(s, 1)$ and $(-1, t) \sim(1, t)$. Let $E$ be the space obtained by imposing on the disjoint union of $T^{2}$ and $K^{2}$ the equivalence relation that $[s, t] \in T^{2}$ is equivalent to $\left[s^{\prime}, t^{\prime}\right] \in K^{2}$ if and only if $s=s^{\prime}$ and $t=t^{\prime}=0$. Projection on the first factor gives a nontrivial bundle $\mathfrak{F}=\left(E, p, S^{1} ; S^{1} \vee S^{1}\right)$ such that $\left(\mathfrak{F}, \mathfrak{F}_{0}\right)$ is a bundle pair which has trivial complement because $\mathfrak{F}_{0}^{c}$ is just the projection of $T^{2}$ to $S^{1}$.

We will generalize this example, for later use, as follows. Let $\mathfrak{F}_{0}=$ $\left(E_{0}, p, B ; Y_{0}\right)$ be a nontrivial bundle with a cross-section $\sigma: B \rightarrow E_{0}$, that is, $p \sigma: B \rightarrow B$ is the identity map. For instance, $\mathfrak{F}_{0}$ could be the tangent sphere bundle of a differentiable manifold $B$ that 
is of Euler characteristic zero so that it has a nonvanishing vector field. To define $E$, take the disjoint union of $E_{0}$ and $B \times Q$, for some space $Q$, and choose $q_{0} \in Q$. The space $E$ is obtained by identifying $\sigma(b)$ and $\left(b, q_{0}\right)$ for each $b \in B$ and extending $p$ to $E$ by projecting $B \times Q$ to $B$. Thus we obtain the bundle pair $\left(\mathfrak{F}, \mathfrak{F}_{0}\right)=$ $\left(\left(E, E_{0}\right), p, B ;\left(Y_{0} \vee Q, Y_{0}\right)\right)$ which has trivial complement because $\mathfrak{F}_{0}^{c}$ is the projection of $B \times Q$ onto $B$.

A finite polyhedron $X$ is 2-dimensionally connected if for any two maximal simplices $s, s^{\prime}$ of $X$ of dimension at least two, there is a set $s_{1}, \ldots, s_{k}$ of maximal simplexes such that $s=s_{1}, s^{\prime}=s_{k}$ and the dimension of $s_{j} \cap s_{j+1}$ is at least one, for each $j=1, \ldots, k-1$. By a fiber deformation of a bundle $\mathfrak{F}=(E, p, B ; Y)$ we mean a map that is fiber homotopic to the identity map of $E$.

Lemma 5.1. Let $\left(\mathfrak{F}, \mathfrak{F}_{0}\right)=\left(\left(E, E_{0}\right), p, B ;\left(Y, Y_{0}\right)\right)$ be a bundle pair with trivial complement where $E, B$ and $Y$ are connected finite polyhedra, $E_{0}$ is a closed, locally contractible subset of $E$ and $Y_{0}^{c}$ is a 2-dimensionally connected polyhedron. Then there exists a fiber deformation $g: E \rightarrow E$ such that Fix $(g)=E_{0}$.

Proof. Since $Y_{0}^{c}$ is 2-dimensionally connected, by Theorem 4.1 of [10], there is a deformation $g^{*}: Y_{0}^{c} \rightarrow Y_{0}^{c}$ such that Fix $\left(g^{*}\right)=$ $\operatorname{bd}\left(Y_{0}\right)$ where bd denotes the boundary. By hypothesis, there is a homeomorphism $\phi: B \times Y_{0}^{c} \rightarrow E_{0}^{c}$ such that $p_{c} \phi=\pi$. Define $g_{0}: E_{0}^{c} \rightarrow E_{0}^{c}$ by $g_{0}(e)=\phi\left(\left(i d \times g^{*}\right)\left(\phi^{-1}(e)\right)\right)$. Note that $g_{0}$ is a fiber deformation because $g^{*}$ is a deformation and that $\operatorname{Fix}\left(g_{0}\right)=$ $\mathrm{bd}\left(E_{0}\right)$. Extending $g_{0}$ to $g: E \rightarrow E$ by letting $g$ be the identity on $E_{0}$ completes the proof.

Theorem 5.1. Let $\mathfrak{F}=(E, p, B ; Y)$ be a bundle where $E, B$ and $Y$ are connected finite polyhedra and $B$ is 2-dimensionally connected. Suppose a closed, locally contractible subset $A$ of $E$ is a bundle subset of $E$ such that, for each component $p(A)_{j}$ of $p(A)$, the bundle pair $\left(p^{-1}\left(p(A)_{j}\right), A_{j}\right)$ has trivial complement and $Y_{j}^{c}$ is a 2-dimensionally connected polyhedron. Then there exists a fiber deformation $g: E \rightarrow$ E such that Fix $(g)=A$.

Proof. Since $B$ is 2-dimensionally connected, by Theorem 4.1 of [10], there is a deformation $\bar{g}: B \rightarrow B$ such that $\operatorname{Fix}(\bar{g})=p(A)$. Moreover, we can see from the proof of Theorem 3.1 of [8] that $\bar{g}$ can be made homotopic to the identity map of $B$ by means of a homotopy $\bar{H}: B \times I \rightarrow B$ with $\bar{H}(b, 0)=b$ such that, if $b \in p(A)$, then $\bar{H}(b, t)=b$ for all $t$. By the homotopy lifting property, there is a homotopy $U: E \times I \rightarrow E$ lifting $\bar{H}$ where, for each $e \in E, U(e, 0)=e$ and, if $p(e) \in p(A)$, then $U(e, t)=e$ for all $t$. Define $u: E \rightarrow E$ 
by $u(e)=U(e, 1)$, then the restriction of the fiber deformation $u$ to $p^{-1}(p(A))$ is the identity map and $p u=\bar{g} p$. Since the hypotheses of Lemma 5.1 are satisfied for the bundle pair $\left(p^{-1}\left(p(A)_{j}\right), A_{j}\right)$, there is a fiber homotopy $H_{j}: p^{-1}\left(p(A)_{j}\right) \times I \rightarrow p^{-1}\left(p(A)_{j}\right)$ such that $H_{j}(e, 0)=e$ and, for $g_{j}$ defined by $g_{j}(e)=H_{j}(e, 1)$, we have $\operatorname{Fix}\left(g_{j}\right)=A_{j}$. Let $H: E \times\{0\} \cup p^{-1}(p(A)) \times I \rightarrow E$ be defined by $H(e, 0)=u(e)$ and $H(e, t)=H_{j}(e, t)$ if $p(e) \in p(A)_{j}$. By the fiber homotopy extension theorem, we can extend $H$ to a homotopy $H: E \times I \rightarrow E$ such that $p H(e, t)=\bar{g} p(e)$. Defining a fiber deformation $g: E \rightarrow E$ by $g(e)=H(e, 1)$ we note that since $p g=\bar{g} p$ where $\operatorname{Fix}(\bar{g})=p(A)$, then $\operatorname{Fix}(g) \subseteq p^{-1}(p(A))$ and therefore $\operatorname{Fix}(g)=A$.

Lemma 5.1 implies that, for the examples

$$
\left(\mathfrak{F}, \mathfrak{F}_{0}\right)=\left(\left(E, E_{0}\right), p, B ;\left(Y_{0} \vee Q, Y_{0}\right)\right)
$$

above, constructed from a nontrivial bundle $\mathfrak{F}_{0}=\left(E_{0}, p, B ; Y_{0}\right)$ with a cross-section and the trivial bundle obtained by projecting $B \times Q$ onto $B$, for any connected finite polyhedron $Q$, there is a fiber deformation $g$ such that $\operatorname{Fix}(g)=E_{0}$ provided that $Y_{0}$ is 2-dimensionally connected. The specific example obtained from the Klein bottle and torus is easily modified to illustrate this class of examples. Let $E_{0}$ be the cartesian product of the Klein bottle and the unit iterval $I$ represented as the quotient space of $[-1,1] \times[-1,1] \times I$ under the equivalence relation $(s,-1, r) \sim(-s, 1, r)$ and $(-1, t, r) \sim(1, t, r)$. Then projection on the first factor gives a nontrivial bundle $\mathfrak{F}_{0}=$ $\left(E_{0}=K^{2} \times I, p, S^{1} ; S^{1} \times I\right)$. Now the fiber $Y_{0}=S^{1} \times I$ is 2dimensionally connected. Again represent the torus $T^{2}=S^{1} \times S^{1}$ as $[-1,1] \times[-1,1]$ under the equivalence relation $(s,-1) \sim(s, 1)$ and $(-1, t) \sim(1, t)$. Let $E$ be the space obtained by imposing on the disjoint union of $T^{2}$ and $K^{2} \times I$ the equivalence relation that $[s, t] \in T^{2}$ is equivalent to $\left[s^{\prime}, t^{\prime}, r\right] \in K^{2} \times I$ if and only if $s=s^{\prime}$ and $t=t^{\prime}=r=0$. Projection on the first factor gives a nontrivial bundle $\mathfrak{F}=\left(E, p, S^{1} ;\left(S^{1} \times I\right) \vee S^{1}\right)$ such that $\left(\mathfrak{F}, \mathfrak{F}_{0}\right)$ is a bundle pair which has trivial complement because $\mathfrak{F}_{0}^{c}$ is just the projection of $T^{2}$ to $S^{1}$. Therefore, there is a fiber deformation $g: E \rightarrow E$ with $\operatorname{Fix}(g)=K^{2} \times I$.

Corollary 5.1. Let $\mathfrak{F}=(E, p, B ; Y)$ be a bundle where $E, B$ and $Y$ are connected finite polyhedra and $B$ is 2-dimensionally connected. Suppose a closed, locally contractible subset $A$ of $E$ is a bundle subset of $E$ such that each component $p(A)_{j}$ of $p(A)$ is contractible and $Y_{j}^{c}$ is a 2-dimensionally connected polyhedron for all $j$. Then there exists a fiber deformation $g: E \rightarrow E$ such that Fix $(g)=A$. 
Theorem 5.2. Let $\mathfrak{F}=(E, p, B ; Y)$ be a bundle where $E, B$ and $Y$ are 2-dimensionally connected finite polyhedra. Suppose $A$ is a closed subset of $E$ such that $p(A)=\left\{b_{1}, \ldots, b_{r}\right\}$, then there exists a fiber deformation $g: E \rightarrow E$ such that Fix $(g)=A$.

Proof. As in the proof of Theorem 5.1, there is a deformation $\bar{g}: B \rightarrow B$ such that $\operatorname{Fix}(\bar{g})=p(A)$ and a fiber deformation $u$ : $E \rightarrow E$ such that $p u=\bar{g} p$ which is the identity map on $p^{-1}(p(A))$. By Theorem 4.1 of [10], for each $j=1, \ldots, r$ there is a homotopy $H_{j}: p^{-1}\left(b_{j}\right) \times I \rightarrow p^{-1}\left(b_{j}\right)$ such that $H_{j}(e, 0)=e$ and, for $g_{j}$ defined by $g_{j}(e)=H_{j}(e, 1)$ we have $\operatorname{Fix}\left(g_{j}\right)=A \cap p^{-1}\left(b_{j}\right)$. Let

$$
H:(E \times\{0\}) \cup\left(\bigcup_{j=1}^{r} p^{-1}\left(b_{j}\right) \times I\right) \rightarrow E
$$

be defined by $H(e, 0)=u(e)$ and $H(e, t)=H_{j}(e, t)$ for $e \in p^{-1}\left(b_{j}\right)$. Extend $H$ to $G: E \times I \rightarrow E$ by the fiber homotopy extension theorem and set $g(e)=G(e, 1)$ to define the fiber deformation such that $\operatorname{Fix}(g)=A$.

\section{References}

[1] Allaud, G. and Fadell, E. A fiber homotopy extension theorem, Trans. Amer. Math. Soc. 104 (1962), 239 - 251.

[2] Hatcher, A. Algebraic Topology, Cambridge Univ. Press, 2002.

[3] Heath, P. A Nielsen type number for fibre preserving maps, Top, Appl. 53 (1993), 19 - 35.

[4] Heath, P., Keppelmann, E. and Wong, P. Addition formulae for Nielsen numbers and for Nielsen type numbers of fibre preserving maps, Top. Appl. 67 1995, 133 - 157.

[5] Husemoller, D., Fibre Bundles, McGraw-Hill, 1966.

[6] Jiang, B. On the least number of fixed points, Amer. J. Math. 102 (1980), 749 - 763.

[7] Jiang, B. Lectures on Nielsen Fixed Point Theory, Contemp. Math. 14 (1983), American Math. Soc.

[8] Schirmer, H. Fixed point sets of polyhedra, Pacific J. Math. 52 (1974), $221-225$.

[9] Schirmer, H. A relative Nielsen number, Pacific J. Math. 122 (1986), 459 - 473. 
[10] Schirmer, H. Fixed point sets in a prescribed homotopy class, Top. Appl. 37 (1990), 153 - 162.

[11] Soderlund, C. Fixed point sets of maps homotopic to a given map, Fixed Point Theory Appl. 2006, Article ID 46052, 1 - 20.

[12] Steenrod, N. The Topology of Fibre Bundles, Princeton Univ. Press, 1951.

[13] You, C. Fixed point classes of a fiber map, Pacific J. Math. 100 (1982), $217-241$. 